\title{
Riboflavin prophylaxis in pediatric and adolescent migraine
}

\author{
Maria Condò · Annio Posar · Annalisa Arbizzani · \\ Antonia Parmeggiani
}

Received: 24 April 2009/Accepted: 10 July 2009/Published online: 1 August 2009

(C) Springer-Verlag 2009

\begin{abstract}
Migraine is a common disorder in childhood and adolescence. Studies on adults show the effectiveness and tolerability of riboflavin in migraine prevention, while data on children are scarce. This retrospective study reports on our experience of using riboflavin for migraine prophylaxis in 41 pediatric and adolescent patients, who received 200 or $400 \mathrm{mg}$ /day single oral dose of riboflavin for 3, 4 or 6 months. Attack frequency and intensity decreased $(P<0.01)$ during treatment, and these results were confirmed during the follow-up. A large number of patients $(77.1 \%)$ reported that abortive drugs were effective for controlling ictal events. During the follow-up, $68.4 \%$ of cases had a $50 \%$ or greater reduction in frequency of attacks and $21.0 \%$ in intensity. Two patients had vomiting and increased appetite, respectively, most likely for causes unrelated to the use of riboflavin. In conclusion, riboflavin seems to be a well-tolerated, effective, and low-cost prophylactic treatment in children and adolescents suffering from migraine.
\end{abstract}

Keywords Riboflavin - Migraine - Headache ·

Treatment $\cdot$ Childhood $\cdot$ Adolescence

\section{Introduction}

Migraine is a common neurological disorder in childhood and adolescence; it has a prevalence of 3\% in infancy/early childhood, increasing up to $23 \%$ in adolescence [1]. If

M. Condò · A. Posar · A. Arbizzani · A. Parmeggiani ( $\square$ ) Child Neurology and Psychiatry Unit,

Department of Neurological Sciences,

University of Bologna, Via Ugo Foscolo 7, 40123 Bologna, Italy

e-mail: antonia.parmeggiani@unibo.it intensity and frequency of migraine become excessive, they may cause a relevant impairment of quality of life. In such cases, a prophylactic treatment is necessary to avoid excessive drug intake, and improve the quality of life [2].

Many drugs used as migraine prophylaxis in adults have not been adequately tested on children, for whom symptomatic drugs are usually preferred.

Riboflavin is the precursor of flavin mononucleotide and flavin adenine dinucleotide which are involved in mitochondrial electron transport chain. Administering of riboflavin in migraine treatment is based on the hypothesis of a deficient mitochondrial energy reserve as a causal factor in migraine [3, 4], and on the findings of its effectiveness at high doses (100-200 mg/day) in the treatment of patients suffering from classic mitochondriopathies [5-8]. Literature reports four studies (two open trials, and two randomized and controlled) about riboflavin high doses (400 mg/day) that suggest its efficacy and tolerability for preventing migraine in adults [9-12]. A recent randomized, controlled trial reports that riboflavin is not so effective in children [13].

At present, there are no further studies about riboflavin prophylaxis in pediatric migraine [14].

We have undertaken this retrospective study to evaluate the effectiveness and tolerability of riboflavin in children and adolescents with resistant migraine.

\section{Patients and methods}

We have examined retrospectively 41 outpatients (16 males, 25 females) who were treated in the Child Neurology and Psychiatry Unit of the Department of Neurological Sciences of the University of Bologna from February 2002 to December 2007. Mean age was 13 years, 7 months 
(range 8 years, 11 months-18 years, 10 months). Mean follow-up from the first to the last observation was 1 year, 6 months. Patients received the following diagnoses, according to the criteria of the International Headache Society [15]: migraine without aura (24), migraine with aura (8), migraine without aura and frequent episodic tension-type headache (7), basilar-type migraine (1), benign paroxysmal vertigo of childhood and frequent episodic tension-type headache (1). Inclusion criteria were: resistant migraine with failure of previous prophylactic therapy (flunarizine, magnesium, and pizotifen); at least three moderate-severe or two severe attacks per month; and no prophylactic treatment for 3 months before the study. Exclusion criteria were: organic or psychiatric diseases. Intensity of attacks was functionally evaluated on a 3-point scale: "1" for mild headache that allowed for carrying out an activity as usual, " 2 " for moderate headache that slackened an activity without stopping it, and " 3 " for severe headache that stopped an activity, and needed repose or sleep.

Our patients were treated with a dose of either 200 or $400 \mathrm{mg} /$ day chosen at random, in galenic preparation, at breakfast [5, 9-11]. Each patient kept a journal to record: number and intensity of attacks, concomitant symptoms, and symptomatic medication with its degree of efficacy. Informed consent was obtained from all patients' parents. Riboflavin was administered for 3, 4, or 6 months.

We have thus subdivided our study in the following 3-month periods: a baseline period without prophylactic medications (Phase 1), riboflavin treatment (Phase 2), and follow-up after the suspension of riboflavin (Phase 3). We have also considered two more phases in patients who assumed riboflavin for 4 or 6 months, and evaluated the last 3 months of therapy: Phase 2a (2nd, 3rd, and 4th month) and Phase 2b (4th, 5th, and 6th month), respectively (Fig. 1). We compared attack frequency, intensity, and symptomatic therapy efficacy in Phases 2, 2a, $2 \mathrm{~b}$, and 3 with Phase 1 . Primary end-point of the study was the reduction of mean attack frequency; secondary endpoints were the reduction of mean headache intensity and the increase of symptomatic therapy responsiveness. Responders were patients with a frequency and/or intensity reduction of at least $50 \%$, while semi-responders showed a $25-50 \%$ reduction. We have also considered: riboflavin effect on aura symptoms; correlation between riboflavin efficacy and sex, age (under or over 12 years), different migraine types, and age of headache onset (under or over 10 years).

We have utilized Wilcoxon sign rank and Fisher exact test for statistical analysis.

\section{Results}

Twenty-one patients (51.2\%) took $200 \mathrm{mg} / \mathrm{day}$ of riboflavin, 20 patients (48.8\%) $400 \mathrm{mg} /$ day. Forty patients (97.6\%) took riboflavin regularly for 3 months at least, concluding Phase 2; one dropped out due to vomiting. Fourteen patients $(35.0 \%)$ continued therapy for 4 months, $11(27.5 \%)$ for 6 months. Thirty-eight patients $(95.0 \%)$ completed Phase 3; for two patients we do not have a 3-month follow-up because they dropped out of the study (Fig. 1).

With regard to the primary end-point, our data show a significant reduction of mean attack frequency in Phase 2 compared with baseline $(21.7 \pm 13.7$ vs. $13.2 \pm 11.8$; $P<0.01)$; this was confirmed during the follow-up in Phase 3 (21.9 \pm 14 vs. $8 \pm 9 ; P<0.01)$. Mean frequency decreased even more in Phase $2 \mathrm{a}(23.4 \pm 12.2$ vs. $8.9 \pm 9.4 ; P<0.01)$, while the decrease was not

Fig. 1 Study flow-chart

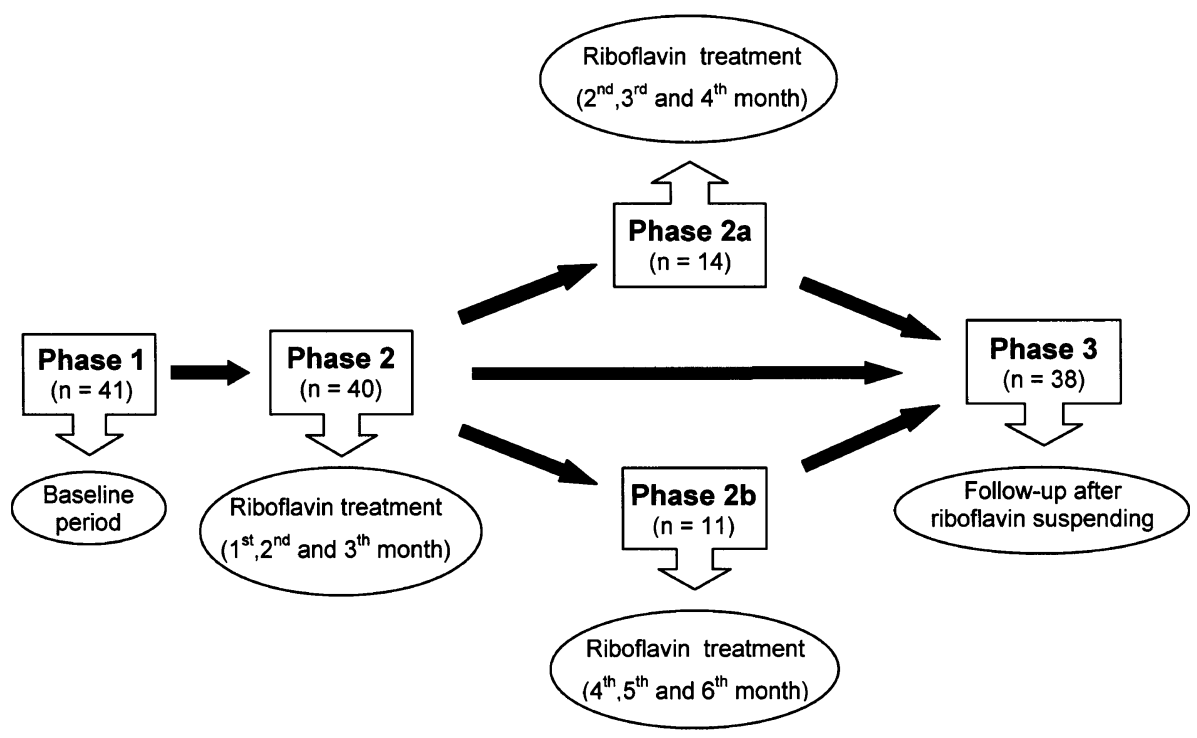


significant in Phase 2b $(19.3 \pm 13.4$ vs. $11.4 \pm 9.6$; $P>0.05$ ) (Fig. 2).

Mean headache intensity decreased from $2( \pm 0.5$; Phase 1) to $1.6( \pm 0.8 ; P<0.01)$ in Phase 2 , and continued to decrease in Phase 3 ( $2 \pm 0.5$ vs. $1.4 \pm 0.9 ; P<0.01)$; whereas there was no significant reduction in headache intensity both in Phase $2 \mathrm{a}(1.8 \pm 0.4$ vs. $1.3 \pm 0.9$; $P>0.05)$ and in Phase $2 \mathrm{~b}(2.1 \pm 0.4$ vs. $1.9 \pm 0.8$; $P>0.05)$ (Fig. 3).

Five patients $(12.5 \%)$ did not use symptomatic drugs during Phases 2 and 3, due to the complete remission of headache or the mildness and infrequent occurrence of migraine attacks; while 27 out of 35 patients $(77.1 \%)$ reported that symptomatic drugs (e.g., ibuprofen, paracetamol, nimesulide, ketoprofen, acetylsalicylic acid, metamizole sodium, noramidopyrine, zolmitriptan, sumatriptan, and piroxicam) were more effective during riboflavin treatment.

Seventeen patients (42.5\%) showed at least a $50 \%$ reduction in attack frequency (responders) in Phase 2, while $7(17.5 \%)$ showed a $25-50 \%$ reduction (semiresponders) in the same phase. The rate of frequency responders increased to $83.3 \%$ in Phase $2 \mathrm{a}$, decreased to $45.4 \%$ in Phase 2b, and was $68.4 \%$ in Phase 3. Semiresponders rate was $0 \%$ in Phase $2 \mathrm{a}, 18.2 \%$ in $2 \mathrm{~b}$ and $18.4 \%$ in Phase 3 . With regard to attack intensity, the responder rate was $21.0 \%$ in Phase 3 (Phase 2: $10.0 \% ; 2 \mathrm{a}$ : $21.4 \%$; 2b: $9.1 \%$ ), while the semi-responder rate in the same phase was $26.3 \%$ (Phase 2: $25.0 \% ; 2 \mathrm{a}: 28.6 \% ; 2 \mathrm{~b}$ : $18.2 \%)$. Statistical analysis showed no significant differences between frequency/intensity responders and nonresponders for a 200 or $400 \mathrm{mg} /$ day dose, different migraine types, and age of headache onset. As regards, the correlation between riboflavin efficacy and sex or age, we have found a significant prevalence of males in the intensity-responder group $(P<0.05)$, and of patients under 12 years in the frequency-responder group $(P<0.05)$.

Among the eight patients suffering from migraine with aura, riboflavin had the following effects in all phases: two patients $(25.0 \%)$ no longer had attacks with aura; two patients $(25.0 \%)$ no longer had attacks with aura, or aura symptoms became shorter and less intense; in one patient $(12.5 \%)$ aura intensity and duration decreased considerably.

During the treatment, one patient dropped out because of vomiting probably caused by the unpleasant flavor of the compound; another patient had increased appetite, but without weight gain. Finally, some patients reported a temporary yellow-orange coloration of urine.

\section{Discussion}

While the literature reports positive data concerning migraine treatment with riboflavin in adult patients [9-12], a recent trial has found no significant effects in childhood [13]. We estimated whether the use of this vitamin can provide benefits also for children and adolescents, and to possibly determine the right dose and duration of the therapy.

Our data suggest that riboflavin prophylactic treatment significantly reduces migraine frequency and intensity $(P<0.01)$ during the trial $(3$-month treatment, Phase 2$)$ and also in the follow-up (Phase 3 ). These results are in line
Fig. 2 Mean headache frequency: comparison between Phase 1 and, respectively, Phase $2(P<0.01)$, Phase $2 \mathrm{a}$ $(P<0.01)$, Phase $2 \mathrm{~b}$ $(P>0.05)$, Phase $3(P<0.01)$

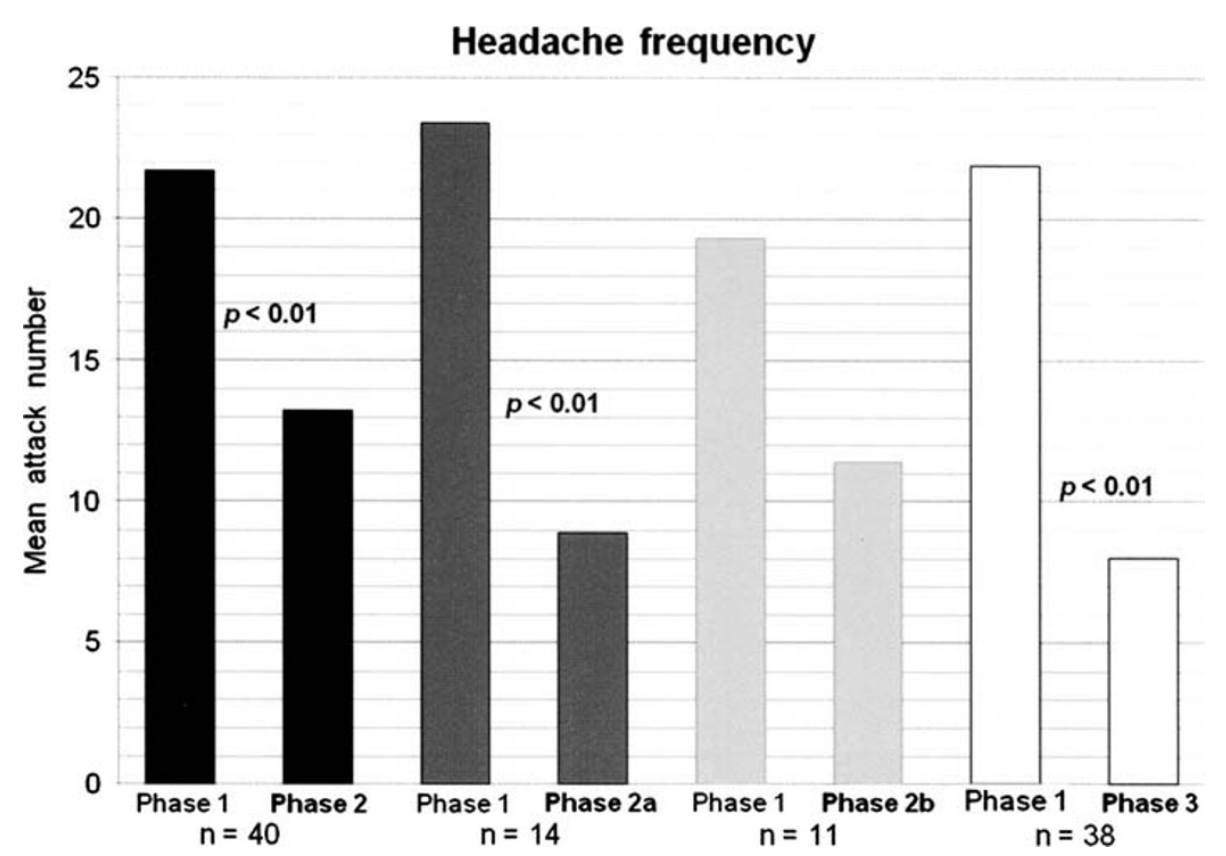


Fig. 3 Mean headache intensity: comparison between Phase 1 and, respectively, Phase $2(P<0.01)$, Phase $2 \mathrm{a}$ $(P>0.05)$, Phase $2 \mathrm{~b}$ $(P>0.05)$, Phase $3(P<0.01)$
Headache intensity

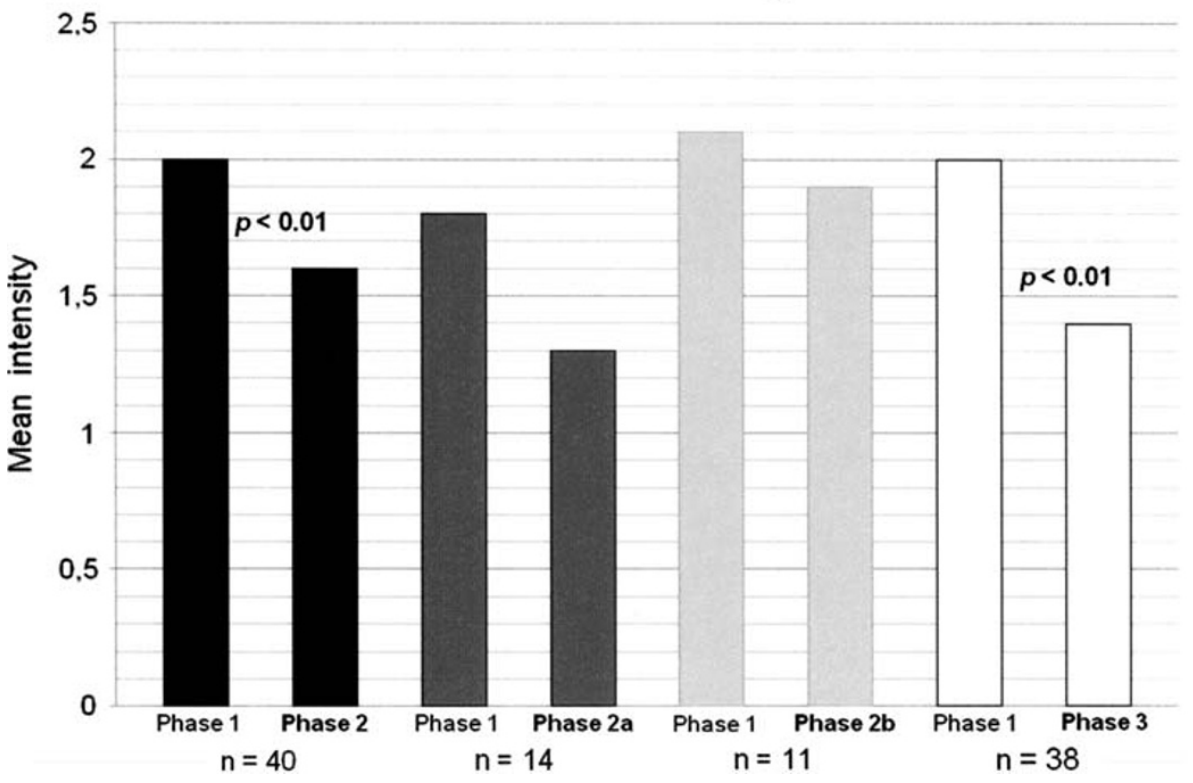

with previous adult studies only with regard to headache frequency, but not to headache intensity [9-11]. In a recent pediatric trial, riboflavin (200 mg daily) was not more effective than placebo for responders in the number of migraine attacks, mean severity per day, days with nausea or vomiting, or number of attacks treated with symptomatic treatment [13]. Our recommendation to parents and patients about an immediate symptomatic pharmacological intervention during riboflavin treatment could have influenced positively the results about headache intensity; in fact in $77.1 \%$ of patients there was an increase of symptomatic therapy responsiveness. The prevalent effect of riboflavin was the reduction of migraine frequency (68.4\% responders, $18.4 \%$ semi-responders, particularly among younger patients, under 12 years: $91.7 \%$ responders). We did not find such significant data for intensity considering all patients; however, our results show an important effect of riboflavin on intensity in males. The gender difference is difficult to explain particularly for prophylactic treatment. Nevertheless, for acute treatment some literature data suggest a higher response rate in boys [16].

We chose a riboflavin dose of 200 or $400 \mathrm{mg} /$ day considering other trials [9-11], the absence of reports on riboflavin toxicity, and finally, the hypothesis that prolonged retention of the vitamin in the intestine can increase the total amount absorbed [17], although riboflavin intestinal absorption is a saturable process [18]. We also tested a $200 \mathrm{mg} /$ day dose, on the basis of dosage utilized in mitochondriopathies [5-8]. Our results confirmed that $200 \mathrm{mg} /$ day of riboflavin may be adequate.

As regards treatment duration, in the Australian trial patients were given riboflavin for 3 months [13], whereas, in our opinion, 4 months seem to be necessary to achieve the best results; in fact, frequency reduction increased up to the 4th month of therapy (Phase 2a), when there was the most elevated rate of responders. The apparent decrease of riboflavin effectiveness in Phase $2 b$ for headache frequency and in Phases $2 \mathrm{a}$ and $2 \mathrm{~b}$ for intensity may be related to the smaller number of patients who reached these phases.

It is also important to underscore that riboflavin was effective in eliminating or reducing the intensity and duration of aura symptoms in five out of eight patients. No data on this topic were reported [9-13].

Our results suggest that riboflavin may be effective for any type of migraine, with any onset age, in childhood and adolescence.

Riboflavin was well tolerated by our patients with an excellent compliance rate: only two patients reported vomiting or increased appetite, probably due to causes not related to the treatment.

We are aware that our results may be open to questioning and discussion because of the placebo response, which has been systematically evaluated in open adult trials for migraine prophylaxis [19], and could be considerably higher in a pediatric study [13]. However, we believe that it would have been unethical to propose a placebo to younger patients with severe and resistant migraine who had come to a specialized university centre for headache treatment and for whom previous therapies had failed. In the pediatric Australian trial [13], the sample was mainly community-based as most of the children were recruited via school newsletters. We suggest that due to this sampling, it is possible that the symptomatology addressed in that trial was less severe than the one we 
observed in our patients, and that as a consequence, the placebo administration was simpler and the results about riboflavin were less encouraging than ours.

Our study has several positive aspects. Sample size is larger than in other studies [9, 11, 20, 21]. Riboflavin is devoid of adverse effects, so it is easy to recommend its use as a therapeutic option to parents of younger patients, who often have reservations about pharmacological therapy. Moreover, its cost is lower than that of most drugs used for migraine prevention.

In conclusion, we suggest that riboflavin might be a safe, well-tolerated, and effective alternative prophylactic treatment for children and adolescents with migraine, while recognizing that randomized controlled studies with a larger number of patients and other standard efficacy parameters could be useful to confirm our results.

Conflict of interest None.

\section{References}

1. Lewis D, Ashwal S, Hershey A, Hirtz D, Yonker M, Silberstein S (2004) Practice parameter: pharmacological treatment of migraine headache in children and adolescents: report of the American Academy of Neurology Quality Standards Subcommittee and the Practice Committee of the Child Neurology Society. Neurology 63:2215-2224

2. Silberstein SD (2000) Practice parameter: evidence-based guidelines for migraine headache (an evidence-based review): report of the Quality Standards Subcommittee of the American Academy of Neurology. Neurology 55:754-762

3. Barbiroli B, Montagna P, Cortelli P, Funicello R, Iotti S, Monari L, Pierangeli G, Zaniol P, Lugaresi E (1992) Abnormal brain and muscle energy metabolism shown by $31 \mathrm{P}$ magnetic resonance spectroscopy in patients affected by migraine with aura. Neurology 42:1209-1214

4. Montagna P, Cortelli P, Monari L, Pierangeli G, Parchi P, Lodi R, Iotti S, Frassineti C, Zaniol P, Lugaresi E et al (1994) 31Pmagnetic resonance spectroscopy in migraine without aura. Neurology 44:666-669

5. Antozzi C, Garavaglia B, Mora M, Rimoldi M, Morandi L, Ursino E, Di Donato S (1994) Late-onset riboflavin-responsive myopathy with combined multiple acyl coenzyme A dehydrogenase and respiratory chain deficiency. Neurology 44:21532158
6. Arts WFM, Scholte HR, Boggard JM, Kerrebijn KF, Luyt-Houwen IEM (1983) NADH-CoQ reductase deficient myopathy: successful treatment with riboflavin. Lancet 2:581-582

7. Penn AM, Lee JW, Thuillier P, Wagner M, Maclure KM, Menard MR, Hall LD, Kennaway NG (1992) MELAS syndrome with mitochondrial tRNA (Leu)(UUR) mutation: correlation of clinical state, nerve conduction and muscle $31 \mathrm{P}$ magnetic resonance spectroscopy during treatment with nicotinamide and riboflavin. Neurology 42:2147-2152

8. Scholte HR, Busch HF, Bakker HD, Bogaard JM, Luyt-Houwen IEM, Kuyt LP (1995) Riboflavin-responsive complex I deficiency. Biochim Biophys Acta 1271:75-83

9. Schoenen J, Lenaerts M, Bastings E (1994) High-dose riboflavin as a prophylactic treatment of migraine: results of an open pilot study. Cephalalgia 14:328-329

10. Schoenen J, Jacquy J, Lenaerts M (1998) Effectiveness of highdose riboflavin in migraine prophylaxis. A randomized controlled trial. Neurology 50:466-470

11. Boehnke C, Reuter U, Flach U, Schuh-Hofer S, Einhäupl KM, Arnold G (2004) High-dose riboflavin treatment is efficacious in migraine prophylaxis: an open study in a tertiary care centre. Eur J Neurol 11:475-477

12. Maizels M, Blumenfeld A, Burchette R (2004) A combination of riboflavin, magnesium, and feverfew for migraine prophylaxis: a randomized trial. Headache 44:885-890

13. MacLennan SC, Wade FM, Forrest KM, Ratanayake PD, Fagan E, Antony J (2008) High-dose riboflavin for migraine prophylaxis in children: a double-blind, randomized, placebo-controlled trial. J Child Neurol 23:1300-1304

14. Tepper SJ (2008) Complementary and alternative treatments for childhood headaches. Curr Pain Headache Rep 12:379-383

15. Headache Classification Sub-Committee of the International Headache Society (2004) The International Classification of Headache Disorders, 2nd edn. Cephalalgia 24(Suppl 1):9-160

16. Lewis DW, Kellstein D, Dahl G, Burke B, Frank LM, Toor S, Northam RS, White LW, Lawson L (2002) Children's ibuprofen suspension for the acute treatment of pediatric migraine. Headache 42:780-786

17. Levy G, Mosovich LL, Allen JE, Yaffe SJ (1972) Biliary excretion of riboflavin in man. J Pharm Sci 61:143-144

18. Zempleni J, Galloway JR, McCormick DB (1996) Pharmacokinetics of orally and intravenously administered riboflavin in healthy humans. Am J Clin Nutr 63:54-66

19. Van der Kuy PH, Lohman JJ (2002) A quantification of the placebo response in migraine prophylaxis. Cephalalgia 22: 265-270

20. Pakalnis A, Kring D, Meier L (2007) Levetiracetam prophylaxis in pediatric migraine-an open-label study. Headache 47: $427-430$

21. Miano S, Parisi P, Pelliccia A, Luchetti A, Paolino MC, Villa MP (2008) Melatonin to prevent migraine or tension-type headache in children. Neurol Sci 29:285-287 